\title{
LncRNA: An all-rounder in rheumatoid arthritis
}

\author{
Junyu Liang, Weiqian Chen, Jin Lin \\ Division of Rheumatology, First Affiliated Hospital, College of Medicine, \\ Zhejiang University, Hangzhou, Zhejiang Province, China
}

Address for Correspondence: Dr. Jin Lin, Division of Rheumatology, First Affiliated Hospital, College of Medicine, Zhejiang University, Hangzhou, 310003 Zhejiang Province, China E-mail: linjinzju@zju.edu.cn

\begin{tabular}{|l|}
\hline Access this article online \\
\hline $\begin{array}{l}\text { Website: } \\
\text { www.intern-med.com }\end{array}$ \\
\hline $\begin{array}{l}\text { DOI: } \\
\text { 10.2478/jim-2019-0002 }\end{array}$ \\
\hline Quick Response Code: \\
\hline \\
\\
\\
\\
\hline
\end{tabular}

\section{ABSTRACT}

Rheumatoid arthritis (RA) is a chronic autoimmune disease and is supposed to have both genetic and environmental backgrounds. Plenty of studies have demonstrated the roles of long non-coding RNAs (IncRNAs) in the initiation and development of RA. Numerous IncRNAs have been found to be dysregulated in RA and to be correlated with disease activity of RA, which indicates potential diagnostic roles of IncRNAs. In addition to working as biomarkers for RA, IncRNAs participate in many specific pathological processes including inflammation, aberrant proliferation, migration, invasion and apoptosis. Further screenings and researches are required to validate the clinical potentials of IncRNAs as diagnostic and therapeutic targets in RA.

Key words: rheumatoid arthritis, IncRNA, inflammation, tumor-like behavior

\section{INTRODUCTION}

Rheumatoid arthritis (RA) is a chronic autoimmune disease featuring persistent synovitis in the joints and systemic inflammation. ${ }^{[1]}$ Approximately $1 \%$ of the worldwide population is affected by $\mathrm{RA}$, making it one of the most prevalent autoimmune diseases nowadays. ${ }^{[2]} \mathrm{RA}$ can lead to functional decline, disability and loss of productivity as a consequence of joint deformity and pain..$^{[3]}$ Initiation and development of RA is supposed to be influenced by both genetic and environmental factors. It has been postulated that a high-risk genetic background, together with epigenetic elements and environmental exposures, leads to a cascade of events initiating RA. ${ }^{[4]} \mathrm{A}$ significant portion of RA patients remain unremitted despite progress in treatment, ${ }^{[5,6]}$ making it crucial to dig deeper into the molecular mechanisms of RA so as to identify the potential therapeutic target for RA.

Previously recognized as "junk" elements, non-coding RNAs (ncRNAs) and their roles in various biological processes have been widely studied in recent years. ${ }^{[7]}$ The ncRNAs involved in epigenetic mechanisms are divided into two principal subgroups: the long ncRNAs (lncRNAs) with more than 200 nucleotides and small ncRNAs with less nucleotides. ${ }^{[8]}$ And according to the position relative to protein-coding genes, lncRNAs can be further classified into pseudogene

lncRNAs, antisense lncRNAs, enhancer RNAs, intronic lncRNAs, and long intergenic noncoding RNAs. ${ }^{[9]}$ To date, thousands of lncRNAs have been identified in human body, and have been shown to control every level of the gene expression. Functions assigned to lncRNAs include transcriptional interference, initiation of chromatin remodeling, promoter inactivation by binding to basal transcription factors, activation of accessory protein, activation and transport of transcription factors, oligomerization of activator protein, and epigenetic repression of genes or gene clusters. ${ }^{[10]}$ Accumulating evidence has shown that $\operatorname{lncRNAs}$ participate in the process of inflammation, aberrant proliferation, migration, invasion and apoptosis, ${ }^{[11-14]}$ and their roles in autoimmune diseases, such as systemic lupus erythematosus (SLE), sjogren syndrome, RA, multiple sclerosis, have also been demonstrated by researchers across the world. ${ }^{[15-19]}$ 
In this review, we intend to identify numerously dysregulated lncRNAs in RA, discuss their potential roles in disease initiation and progression, and seek to sketch the mediating network of lncRNAs.

\section{DYSREGULATED EXPRESSION OF LNCRNAS IN RA}

The most common approaches to identify RA-related lncRNAs are microarray and qPCR (quantitative real-time Polymerase Chain Reaction). The targeted cells range from peripheral blood cells to fibroblast like synoviocytes (FLSs). Yuan and his colleagues targeted the peripheral blood monocyte cells (PBMCs) and identified 2,099 lncRNAs and 2,307 mRNAs that were differentially expressed between the RA patients and healthy controls (HCs). The qPCR results exhibited that the expressions of ENST00000456270 and NR_002838 were significantly increased in the RA patients, whereas the expressions of NR_026812 and uc001zwf.1 were significantly decreased in comparison to those in HCs. ${ }^{[20]}$ Later exploration revealed that the expression of ENST00000456270 was positively correlated with the serum levels of IL-6, TNF- $\alpha$ as well as the Simplified Disease Activity Index (SDAI) of the RA patients. Luo et al. performed microarray on PBMCs of patients diagnosed with RA and HCs. ${ }^{[21]}$ And they recognized a total of 5,045 $\operatorname{lncRNAs}$ (upregulated, 2,410; downregulated, 2,635) and 3,289 mRNAs (upregulated, 1,403; downregulated, 1,886) that were dysregulated in patients with RA. The following lncRNA target prediction revealed the presence of 135 potential lncRNA-mRNA target pairs for the 85 aberrant lncRNAs and 109 aberrant mRNAs.

Zhang et al. used microarray to profile the alterations of lncRNAs in FLSs of tissues isolated from knee joints of RA patients and HCs. ${ }^{[2]} 135$ lncRNAs were differentially expressed between RA FLSs and HCs. The qPCR data revealed that lncRNA ENST00000483588 was up-regulated and three other lncRNAs (ENST00000438399, uc004afb.1, and ENST00000452247) were down-regulated in RA FLSs in comparison to those in HCs. Furthermore, the expression level of ENST00000483588 was found to be positively correlated with the level of serum C-reactive protein (CRP) and SDAI. By calculating the areas under the ROC curves of the above lncRNAs, their potential diagnostic value for RA were also proposed. Similar process was also seen in the research on RA rat model. Jiang et al. compared the expression of lncRNAs in FLSs between the RA model rats induced by Freund's complete adjuvant (FCA) and HCs. ${ }^{[23]} \mathrm{Up}$ to $260 \operatorname{lncRNAs}$ were found to be dysregulated in FCA-induced RA model rats. Of those, six dysregulated lncRNAs including XR_008357,
U75927, MRAK046251, XR_006457, DQ266363 and MRAK003448 were further validated by qRT-PCR.

lncRNAs proven to play a role in other autoimmune diseases or similar biological processes of other diseases could also be studied in RA. Zhang et al. previously identified two novel lncRNAs, lnc0640 and lnc5150, which played a role in the development of SLE and the effect of lnc0640 and lnc5150 in RA was hereby explored. ${ }^{[24]}$ The expression of lnc0640 in PBMCs of RA patients was significantly increased, whereas the expression of lnc5150 was significantly decreased. And the two lncRNAs were found to be significantly associated with the level of serum CRP. In addition, lnc5150 level was as well associated with erythrocyte sedimentation rate (ESR). Certain SNPs within $\operatorname{lnc} 0640$ and lnc5150 were also proved to be linked to RA. LncRNA Gas5 has previously been demonstrated to induce the apoptosis of tumor cells through sponging a number of cancer-related miRNAs, and to exert proapoptotic effects in macrophages and endothelial cells to alleviate atherosclerosis. ${ }^{[25,26]} \mathrm{Li}$ and his colleagues hereby sought to figure out the role of Gas5 in apoptosis of FLSs in RA patients. ${ }^{[27]}$ And apoptosis of FLSs was found to be induced by Gas 5 via activating phosphatidylinositol 3 kinase/protein kinase B (PI3K/AKT) pathway.

\section{DIVERSE ROLES OF LNCRNAS IN PATHOGENESIS OF RA}

\section{Inflammation}

HOTAIR is an lncRNA that has been widely studied in the development of several diseases including various cancers ${ }^{[28]}$ and cardiovascular disease. ${ }^{[2]}$ In 2014 , Song et al. reported a notably elevated expression level of HOTAIR in PBMCs and serum exosome of patients with RA. ${ }^{[30]}$ Besides, overexpressed HOTAIR in exosome help attracting active macrophage into target. However, the expression level of HOTAIR was observed to be significantly decreased in RA FLSs compared to that in HCs. And the enforced expression of HOTAIR alleviated the activation of matrix metalloproteinases MMP-2 and MMP-13. To further validate the role of HOTAIR in RA, Zhang et al. studied the effect of HOTAIR on inflammation in LPS-induced chondrocytes that mimicked the chondrocyte alteration in RA. ${ }^{[31]}$ They identified significant down-regulation of HOTAIR in LPS-induced chondrocytes. Enforced expression of HOTAIR drastically reduced the expression of IL-17 and IL-23, which indicated a protective role of HOTAIR in RA inflammation. Furthermore, the protective mechanism of HOTAIR was found to be associated with the targeted miR-138. An LPS-induced up-regulation of IL- $1 \beta$, TNF- $\alpha$ and p65 was inhibited by HOTAIR overexpression and the effect can be reversed by miR- 
138 mimic transfection. Moreover, the nuclear transfer of p65 was notably inhibited by the overexpression of HOTAIR, which was also partly reversed by miR-138 mimic transfection. Similar results were observed in RA rats as well. A HOTAIR-miR-138-nuclear factor- $x \mathrm{~B}(\mathrm{NF}-x \mathrm{~B})$ axis was hereby recognized by going downstream.

LncRNA H19 is located on chromosome 11 in humans and has been proven to play pivotal roles in proliferation, invasion, and metastasis of tumor. ${ }^{[32,33]}$ Stuhlmuller et al. demonstrated that $\mathrm{H} 19$ expression was up-regulated in the synovial tissue (synovial macrophages and fibroblasts in particular) from patients with RA in comparison to HCs. ${ }^{[34]}$ Moreover the expression of $\mathrm{H} 19$ could be induced to a higher level in RA FLSs by starvation regardless of the treatment of TNF- $\alpha$, IL-1 $\beta$ or platelet-derived growth factor-BB (PDGF-BB). And the PI3K and mitogenactivated protein kinase-1/2 (ERK-1/2) pathways were observed to mediate starvation-induced H19 expression. These results suggested that $\mathrm{H} 19$ might promote RA through PI3K and ERK-1/2 pathways. The specific mediating routine in RA remains unexplored.

$\mathrm{Lu}$ and his colleagues observed significantly increased expression level of lncRNAs LOC100652951 and LOC100506036 in T cells from patients with RA compared with that in HCs. ${ }^{[35]}$ Moreover, the expression of LOC100652951 within RA T cells was inversely correlated with the treatment of biologic agents including tumor necrosis factor antagonists like abatacept and tocilizumab. Meanwhile, expression of LOC100506036 was inversely correlated with the use of cyclosporine, which revealed its possible correlation with $\mathrm{Ca}^{2+}$ influx-related gene. Elevated expression level of LOC100506036 was also found in the activated Jurkat cells and silencing LOC100506036 reduced the expression of IFN- $\gamma$. The expression levels of nuclear factor of activated $\mathrm{T}$ cells 1 (NFAT1), which in turn regulates the expression of various cytokines, ${ }^{[36]}$ was drastically decreased after silencing LOC100506036. These findings indicate that LOC10506036 might promote inflammation in T cells of RA patients through activating the transcription of NFAT1.

NT'T is located on chromosome 6q23-q24, and is supposed to exert its function via regulating the nearby genes. ${ }^{[37]}$ A research published in 2018 revealed the role of C/ $\mathrm{EBP} \beta / \mathrm{NTT} / \mathrm{PBOV} 1$ axis in RA. ${ }^{[38]}$ NTT' was found to express in resting human primary monocytes, monocytederived macrophages, and the THP-1 cell line. And C/ $\mathrm{EBP} \beta$ was observed to bind to the promoter of NTT and promote NTT expression. By going downstream, the researchers showed that NTT might enhance the expression of PBOV1, which led to THP-1's cell cycle arrest and differentiation to macrophages, by interacting with hnRNP-U and binding to the promoter of PBOV1. Furthermore, C/EBP $\beta$, NTT, and PBOV1 expression levels within PBMCs were prominently elevated in RA, and were observed to decline after the treatment. Positive correlation between C/EBP $\beta / \mathrm{NTT} / \mathrm{PBOV} 1$ expressions and initial disease activity scores (DAS28) was identified. C/ EBP $\beta$ expression was also found to be positively correlated with SDAI. These results collectively demonstrate that the $\mathrm{C} / \mathrm{EBP} \beta / \mathrm{NTT} / \mathrm{PBOV} 1$ axis is significantly activated in untreated RA, and their expression levels might be linked to a higher inflammatory status.

Spurlock et al. probed into the role of lincRNA-p21 in RA via analyzing patients' blood samples. ${ }^{[39]}$ Lower basal level of lincRNA-p21 and higher basal level of phosphorylated p65 (a NF- $x$ B subunit) were observed in the RA patients compared with those in HCs. MTX was identified to induce the over-expression of linRNA-p21 through a DNAdependent protein kinase catalytic subunit-dependent (DNA-PKsc) mechanism in activated T cells and Jurkat cells. Besides, they also found that MTX reduced the NF$x \mathrm{~B}$ activity and the following pro-inflammatory effect in TNF- $\alpha$-treated cell in a lincRNA-p21-dependent manner. In this case, the elevated level of lincRNA-p21 is supposed to play a protective role in RA.

Yang and his colleagues identified that shikonin, a major active ingredient isolated from zicao, inhibited inflammatory response in FLSs of RA mice through lncRNA-NR024118-SOCS3 pathway. ${ }^{[40]}$ SOCS proteins were reported to be induced by cytokines and were found to subsequently act in a negative feedback loop to inhibit cytokine signal transduction. ${ }^{[41]}$ Treatment of shikonin in mice was found to increase the expression of lncRNANR024118 and SOCS3, and decreased the expression of inflammatory cytokines and MMPs. In addition, shikonin increased acetylation of $\mathrm{H} 3$ at the NR024118 promoter in RA FLSs. Besides, overexpression and silencing of lncRNA-NR024118 increased and decreased the expression of Socs3, respectively. And silencing lncRNANR024118 aborted the protective effect of shikonin, which could be recovered by NR024118 interference. The effect of human homologue of IncRNA-NR024118 demands further research.

The TRAF1-C5 region was previously identified to be associated with RA. ${ }^{[42]}$ Increased level of C5 was found in the inflamed joints of RA patients, and C5-deficient mice was proven to be resistant to the development of collagen induced arthritis. ${ }^{[43,44]}$ Messemaker et al. found that lncRNA C5T1 was associated with the transcription of C5 in the RA patients. ${ }^{[45]}$ In their study, C5T1 lncRNA was found to express predominantly in the nucleus and its expression was correlated positively with C5 mRNA in 
various tissues and in peripheral blood mononuclear cells, which indicated potential transcriptional co-regulation. Furthermore, knockdown of lncRNA C5T1 resulted in the decrease of C5 mRNA levels in FLSs, which indicated the potential role of lncRNA C5T1 in RA.

\section{Proliferation, Migration and Invasion}

Previous studies demonstrated that RA FLSs shared similar features with tumor cells, such as tumor-like migration, invasion, and aberrant proliferation. ${ }^{[46]}$ Increased proliferation, migration and invasion of FLSs has been proven to greatly contribute to RA initiation and progression. ${ }^{[47]}$ In hepatocellular carcinoma, lncRNA ZFAS1 was found to promote cell migration and invasion via sponging miR-150 and inhibiting its tumor-suppressive function, ${ }^{[48]}$ which brought researchers to suspect the role of ZFAS1 in RA. Ye et al. found ZFAS1 expression was increased in RA FLSs compared with that in HCs. ${ }^{[4]}$ Silencing ZFAS1 suppressed RA FLSs' migration and invasion, while overexpression of ZFAS1 exerted the opposite effect. Further research demonstrated that ZFAS1 directly targeted miR-27a, reduced the expression of miR27a and promoted RA FLSs' migration and invasion in a miR-27a-dependent manner.

LncRNA GAPLINC was also reported to promote proliferation, migration, invasion, and metastasis of cancer cells. ${ }^{[50,51]} \mathrm{Mo}$ and his colleagues showed a greater level of expression of GAPLINC in RA FLSs than that in FLSs of patients with traumatic injury. ${ }^{[52]}$ Knocking-down GAPLINC ameliorated the morbid proliferation of RA FLSs, as well as migration and invasion. Production of various cytokines and MMPs such as IL-6, IL-8 and MMP9 was also decreased after the suppression of GAPLINC. Further verification demonstrated that silencing of GAPLINC increased miR-382-5p and miR-575 expression, suggesting GPALINC might promote proliferation, migration and invasion of RA FLSs via sponging miR-382$5 \mathrm{p}$ and miR-575. These results indicated that GAPLINC might promote tumor-like behaviors of RA FLSs in a miR-382-5p-dependent and miR-575-dependent manner.

Aside from the role in inflammation, HOTAIR was also found to influence the proliferation of chondrocytes. Zhang et al. observed the notably increased cell viability and proliferative ability of LPS-induced chondrocytes after enforced expression of HOTAIR. ${ }^{[31]} \mathrm{Ki} 67$ and PCNA, as two proliferation-associated markers, were over-expressed as well. Similar results were found in cartilage tissues of RA rats after subcutaneous injection of LV-HOTAIR.

\section{Apoptosis}

Apoptosis is an important mechanism that regulates tissue composition and homeostasis. ${ }^{[53]}$ In recent years, apoptosis of synovial cells and inflammatory cells has been considered as a therapeutic tool in RA. To date, studies on lncRNAs and apoptosis mainly focus on FLSs.

Urothelial carcinoma associated 1 (UCA1) is a newlyidentified lncRNA located on the chromosome 19p13.12. Recent years have seen many progresses in the study of UCA1. YAN et al. found that UCA1 was closely related to rheumatoid arthritis. ${ }^{[54]}$ In this study, UCA1 and caspase-3 were proven to be highly expressed in HCs compared with those in RA FLSs. In contrast, cell viability was higher in RA FLSs than in HCs. In addition, knock-down of UCA1 decreased the expression of caspase-3 in HCs, and overexpression of UCA1 increased the expression of caspase-3 within RA FLSs. The opposite happened to cell viability under similar interventions. These results led to the conclusion that UCA1 might play a protective role in RA via inducing apoptosis of FLSs. Furthermore, the authors found that UCA1 suppression in HCs could improve the expression of Wnt6, while UCA1 overexpression in RA FLSs could reduce the expression of Wnt6. And the viability of RA FLSs, which was somehow negatively correlated with apoptosis in this case, recovered after they transfected the over-expressed plasmid of Wnt6 into the UCA1-overexpressed RA FLSs. UCA1 influenced cell viability through altering expression of Wnt6.

Up-regulation of MALAT1 is seen in various human cancers and has been proven to be associated with cancer metastasis and recurrence. ${ }^{[5]}$ Pan et al. observed theelevation of apoptotic rate of RA FLSs after the treatment of quercetin. ${ }^{[56]}$ To figure out the molecular mechanism of the effect of quercetin on RA FLSs, alterations of expression of lncRNAs within the cells were analyzed with the application of PCR array and GPCR. And MALAT1 was found to be the most prominently altered lncRNA in RA FLSs. The knockdown of MALAT1 inhibited the apoptosis of the RA FLSs, decreased the expression of caspase- 3 , caspase- 9 and Bax, and increased the expression of the anti-apoptotic Bcl-2 in the cells. The MALAT1 knockdown also promoted the phosphoinositide 3-kinase PI3K/AKT signaling pathway. These findings demonstrated that MALAT1-dependent suppression of the pro-survival PI3K/AKT pathway contributed to FLSs' apoptosis induced by quercetin.

LncRNA Gas5 was previously found to play a role in the apoptosis of tumor cells, macrophages and endothelial cells. ${ }^{[57,58]}$ A study conducted by researchers from Yangzhou revealed the role of Gas5 in apoptosis of FLSs. ${ }^{[59]}$ Expression of lncRNA Gas5 was found to be down-regulated in RA FLSs in comparison to that in HCs. Besides, the treatment of Tan-IIA, which was supposed to ameliorate RA, aggravated the apoptosis of RA FLSs, 
attenuated the viability of RA FLSs and up-regulated Gas5 at the same time. Knockdown of Gas5 afterwards induced lower expression of caspase-3 and caspase-9, ameliorated the apoptosis of RA FLSs, and activated the PI3K/AKT pathway under the treatment of Tan-IIA. Taken together, PI3K/AKT signaling works as a potent pathway of Gas5mediated FLSs' apoptosis.

\section{CONCLUSION}

The difficulties in early identification and treatment of refractory RA urge researchers to further explore the poorlyunderstood pathogenic mechanism of RA. Recent years have seen accumulating evidence for mediating roles of lncRNAs in RA, including their influence on inflammation, aberrant proliferation, migration, invasion and apoptosis. And the diagnostic value of lncRNAs, which has long been ignored, is also revealed by the researchers. The numerously identified lncRNAs, the comprehensive effects, and the various pathways in between indicate a mediating network of lncRNAs in the initiation and development of RA (Figure 1). Compared with that in cancer research, the network in RA leaves many gaps to fill in. Future screenings are demanded to identify more lncRNAs and their effect on RA. Further animal studies and subsequent clinical trials are also required to validate the clinical potentials of lncRNAs as therapeutic targets in RA.

\section{Source of Foundation}

This study is supported in part by the grants from National Natural Science Foundation of China (81701600) and Natural Science Foundation of Zhejiang Province (LQ17H100001 and LGF18H100001).

\section{Conflict of Interest}

None declared.

\section{REFERENCE}

1. Liu R, Zhao P, Tan W, Zhang M. Cell therapies for refractory rheumatoid arthritis. Clin Exp Rheumatol 2018; 36: 911-9.

2. Gibofsky A. Overview of epidemiology, pathophysiology, and diagnosis of rheumatoid arthritis. Am J Manag Care 2012; 18: S295-302.

3. Janssens X, Decuman S, De Keyser F; Belgian Rheumatoid Arthritis Disability Assessment study group. Comparison of the Belgian Rheumatoid Arthritis Disability Assessment and Health Assessment Questionnaires as Tools to Predict the Need for Support Measures in Patients with Rheumatoid Arthritis. PLoS One 2016; 11: e0146688.

4. Angelotti, F, Parma A, Cafaro G, Capecchi R, Alunno A, Puxeddu I. One year in review 2017: pathogenesis of rheumatoid arthritis. Clin Exp Rheumatol 2017; 35: 368-78.

5. Calvo Alen J, Perez T, Romero Yuste S, Ferraz-Amaro I, Alegre Sancho JJ, Pinto Tasende JA, et al. Efficacy and Safety of Combined Therapy With Synthetic Disease-modifying Antirheumatic Drugs in Rheumatoid Arthritis: Systematic Literature Review. Reumatol Clin 2018, pii: S1699258X(18)30175-X.

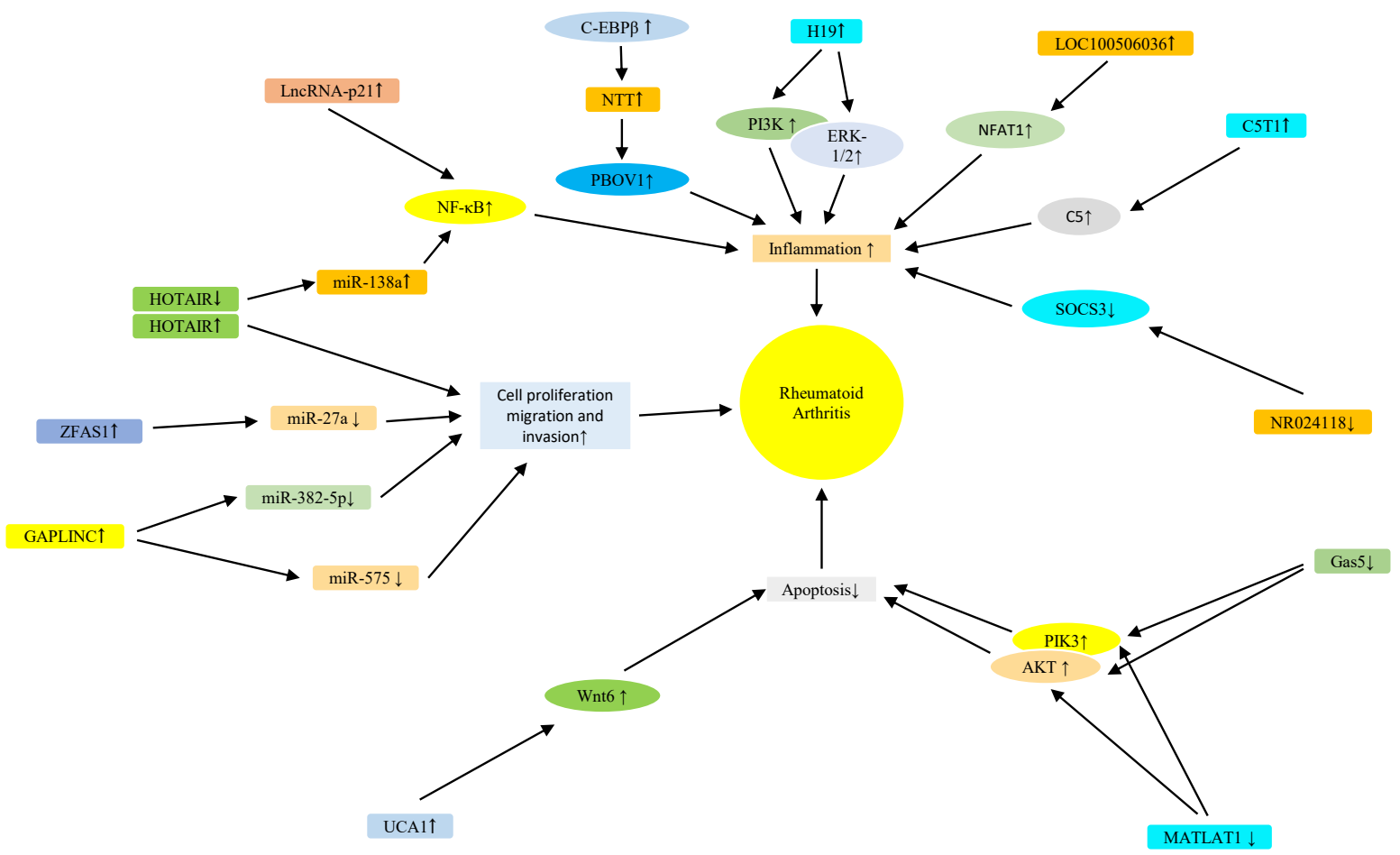

Figure 1 Network of LncRNAs in rheumatoid arthritis. (Synoviocyte: H19, NR024118 (mice), ZFAS1, GAPLINC, UCA1, MALAT1, Gas5 (Synoviocyte and HeLa cell); Monocyte: NTT(Monocyte and THP-1), LncRNA C5T1: T cell and Jurkat cell: LOC100506036, LincRNA-p21; Chondrocyte: HOTAIR) 
6. Buch MH. Defining refractory rheumatoid arthritis. Ann Rheum Dis 2018; 77: 966-9.

7. Wolfien M, Brauer DL, Bagnacani A, Wolkenhauer O. Workflow Development for the Functional Characterization of ncRNAs. Methods Mol Biol 2019; 1912: 111-32.

8. Kolarz B, Majdan M. Epigenetic aspects of rheumatoid arthritis: contribution of non-coding RNAs. Semin Arthritis Rheum 2017; 46: 724-31.

9. Pearson MJ, Jones SW. Review: Long Noncoding RNAs in the Regulation of Inflammatory Pathways in Rheumatoid Arthritis and Osteoarthritis. Arthritis Rheumatol 2016; 68: 2575-83.

10. Ponting, C. P., Oliver, P. L., Reik, W. Evolution and functions of long noncoding RNAs. Cell 2009, 136, 629-641.

11. Ghaffar M, Khodahemmati S, Li J, Shahzad M, Wang M, Wang Y, et al. Long Non-coding RNA LINC01234 Regulates Proliferation, Invasion and Apoptosis in Esophageal Cancer Cells. J Cancer 2018; 9: 4242-9.

12. Dhamija S, Becker AC, Sharma Y, Myacheva K, Seiler J, Diederichs S. LINC00261 and the Adjacent Gene FOXA2 Are Epithelial Markers and Are Suppressed during Lung Cancer Tumorigenesis and Progression. Noncoding RNA 2018; 5: pii: E2.

13. Klec C, Gutschner T, Panzitt K, Pichler M. Involvement of long noncoding RNA HULC (highly up-regulated in liver cancer) in pathogenesis and implications for therapeutic intervention. Expert Opin Ther Targets 2019; 15: 1-10.

14. Cremer S, Michalik KM, Fischer A, Pfisterer L, Jae N, Winter C, et al. Hematopoietic Deficiency of the Long Non-Coding RNA MALAT1 Promotes Atherosclerosis and Plaque Inflammation. Circulation 2018. [Epub ahead of print]

15. Wang J, Peng H, Tian J, Ma J, Tang X, Rui K, et al. Upregulation of long noncoding RNA TMEVPG1 enhances T helper type 1 cell response in patients with Sjogren syndrome. Immunol Res 2016; 64: 489-96.

16. Cardamone G, Paraboschi EM, Solda G, Cantoni C, Supino D, Piccio L, et al. Not only cancer: the long non-coding RNA MALAT1 affects the repertoire of alternatively spliced transcripts and circular RNAs in multiple sclerosis. Hum Mol Genet 2018. [Epub ahead of print]

17. Zhao CN, Mao YM, Liu LN, Li XM, Wang DG, Pan HF. Emerging role of lncRNAs in systemic lupus erythematosus. Biomed Pharmacother 2018; 106: 584-92.

18. Teimuri S, Hosseini A, Rezaenasab A, Ghaedi K, Ghoveud E, Etemadifar $\mathrm{M}$, et al. Integrative Analysis of lncRNAs in Th17 Cell Lineage to Discover New Potential Biomarkers and Therapeutic Targets in Autoimmune Diseases. Mol Ther Nucleic Acids 2018; 12: 393-404.

19. Xu F, Jin $\mathrm{L}$, Jin $\mathrm{Y}$, Nie Z, Zheng $\mathrm{H}$. Long noncoding RNAs in autoimmune diseases. J Biomed Mater Res A 2019; 107: 468-75.

20. Yuan M, Wang S, Yu L, Qu B, Xu L, Liu L, et al. Long noncoding RNA profiling revealed differentially expressed IncRNAs associated with disease activity in PBMCs from patients with rheumatoid arthritis. PLoS One 2017; 12: e0186795.

21. Luo Q, Xu C, Li X, Zeng L, Ye J, Guo Y, et al. Comprehensive analysis of long non-coding RNA and mRNA expression profiles in rheumatoid arthritis. Exp Ther Med 2017; 14: 5965-73.

22. Zhang Y, Xu YZ, Sun N, Liu JH, Chen FF, Guan XL, et al. Long noncoding RNA expression profile in fibroblast-like synoviocytes from patients with rheumatoid arthritis. Arthritis Res Ther 2016; 18: 227.

23. Jiang H, Qin XJ, Li WP, Ma R, Wang T, Li ZQ. LncRNAs expression in adjuvant-induced arthritis rats reveals the potential role of LncRNAs contributing to rheumatoid arthritis pathogenesis. Gene 2016; 593: $131-42$.

24. Zhang TP, Zhang Q, Wu J, Zhao YL, Wang JB, Leng RX, et al. The expression levels of long noncoding RNAs lnc0640 and lnc5150 and its gene single-nucleotide polymorphisms in rheumatoid arthritis patients. J Cell Biochem 2018; 119: 10095-106.

25. Wang Y, Kong D. LncRNA GAS5 Represses Osteosarcoma Cells Growth and Metastasis via Sponging MiR-203a. Cell Physiol Biochem 2018; 45: 844-55.
26. Chen L, Yang W, Guo Y, Chen W, Zheng P, Zeng J, et al. Exosomal lncRNA GAS5 regulates the apoptosis of macrophages and vascular endothelial cells in atherosclerosis. PLoS One 2017; 12: e0185406.

27. Li G, Liu Y, Meng F, Xia Z, Wu X, Fang Y, et al. Tanshinone IIA promotes the apoptosis of fibroblast-like synoviocytes in rheumatoid arthritis by up-regulating lncRNA GAS5. Biosci Rep 2018; 38: pii: BSR20180626.

28. Zhang J, Liu X, You LH, Zhou RZ. Significant association between long non-coding RNA HOTAIR polymorphisms and cancer susceptibility: a meta-analysis. Onco Targets Ther 2016; 9: 3335-43.

29. Carrion K, Dyo J, Patel V, Sasik R, Mohamed SA, Hardiman G, et al. The long non-coding HOTAIR is modulated by cyclic stretch and WNT/ beta-CATENIN in human aortic valve cells and is a novel repressor of calcification genes. PLoS One 2014; 9: e96577.

30. Song J, Kim D, Han J, Kim Y, Lee M, Jin EJ. PBMC and exosome-derived Hotair is a critical regulator and potent marker for rheumatoid arthritis. Clin Exp Med 2015; 15: 121-6.

31. Zhang HJ, Wei QF, Wang SJ, Zhang HJ, Zhang XY, Geng Q, et al. LncRNA HOTAIR alleviates rheumatoid arthritis by targeting miR-138 and inactivating NF- $\kappa B$ pathway. Int Immunopharmacol 2017; 50: 283-90.

32. Ding D, Li C, Zhao T, Li D, Yang L, Zhang B. LncRNA H19/miR-29b-3p/ PGRN Axis Promoted Epithelial-Mesenchymal Transition of Colorectal Cancer Cells by Acting on Wnt Signaling. Mol Cells 2018; 41: 423-35.

33. Sun H, Wang G, Peng Y, Zeng Y, Zhu QN, Li TL, et al. H19 lncRNA mediates 17beta-estradiol-induced cell proliferation in MCF-7 breast cancer cells. Oncol Rep 2015; 33: 3045-52.

34. Stuhlmuller B, Kunisch E, Franz J, Martinez-Gamboa L, Hernandez MM, Pruss A, et al. Detection of oncofetal h19 RNA in rheumatoid arthritis synovial tissue. Am J Pathol 2003; 163: 901-11.

35. Lu MC, Yu HC, Yu CL, Huang HB, Koo M, Tung CH, et al. Increased expression of long noncoding RNAs LOC100652951 and LOC100506036 in T cells from patients with rheumatoid arthritis facilitates the inflammatory responses. Immunol Res 2016; 64: 576-83.

36. Gholijani N, Gharagozloo M, Farjadian S, Amirghofran Z. Modulatory effects of thymol and carvacrol on inflammatory transcription factors in lipopolysaccharide-treated macrophages. J Immunotoxicol 2016; 13: 57-164.

37. Liu AY, Torchia BS, Migeon BR, Siliciano RF. The human NTT gene: identification of a novel 17-kb noncoding nuclear RNA expressed in activated CD4+ T cells. Genomics 1997; 39: 171-84.

38. Yang CA, Li JP, Yen JC, Lai IL, Ho YC, Chen YC, et al. lncRNA NTT/ PBOV1 Axis Promotes Monocyte Differentiation and Is Elevated in Rheumatoid Arthritis. Int J Mol Sci 2018; 19: pii: E2806.

39. Spurlock CF 3rd, Tossberg JT, Matlock BK, Olsen NJ, Aune TM. Methotrexate inhibits NF-kappaB activity via long intergenic (noncoding) RNA-p21 induction. Arthritis Rheumatol 2014; 66: 2947-57.

40. Yang KY, Chen DL. Shikonin Inhibits Inflammatory Response in Rheumatoid Arthritis Synovial Fibroblasts via lncRNA-NR024118. Evid Based Complement Alternat Med 2015; 631737.

41. Alexander, W. S. Suppressors of cytokine signalling (SOCS) in the immune system. Nat Rev Immunol 2012; 2:410-416.

42. Kurreeman FA, Padyukov L, Marques RB, Schrodi SJ, Seddighzadeh M, Stoeken-Rijsbergen G, et al. A candidate gene approach identifies the TRAF1/C5 region as a risk factor for rheumatoid arthritis. PLoS Med 2007; 4: e278.

43. Trouw LA, Pickering MC, Blom AM. The complement system as a potential therapeutic target in rheumatic disease. Nature Reviews Rheumatology 2017; 13: 538-47.

44. Wang Y, Kristan J, Hao L, Lenkoski CS, Shen Y, Matis LA. A role for complement in antibody-mediated inflammation: C5-deficient DBA/1 mice are resistant to collagen-induced arthritis. J Immunol 2000; 164: 4340-7.

45. Messemaker TC, Frank-Bertoncelj M, Marques RB, Adriaans A, Bakker AM, Daha N, et al. A novel long non-coding RNA in the rheumatoid arthritis risk locus TRAF1-C5 influences C5 mRNA levels. Genes Immun 2016; 17: 85-92. 
46. Liu Y, Pan YF, Xue YQ, Fang LK, Guo XH, Guo X, et al. uPAR promotes tumor-like biologic behaviors of fibroblast-like synoviocytes through PI3K/Akt signaling pathway in patients with rheumatoid arthritis. Cell Mol Immunol 2018; 15: 171-81.

47. Guo X, Zhang D, Zhang X, Jiang J, Xue P, Wu C, et al. Dyrk1A promotes the proliferation, migration and invasion of fibroblast-like synoviocytes in rheumatoid arthritis via down-regulating Spry2 and activating the ERK MAPK pathway. Tissue Cell 2018; 55: 63-70.

48. Li T, Xie J, Shen C, Cheng D, Shi Y, Wu Z, et al. Amplification of Long Noncoding RNA ZFAS1 Promotes Metastasis in Hepatocellular Carcinoma. Cancer Res 2015; 75: 3181-91.

49. Ye Y, Gao X, Yang N. LncRNA ZFAS1 promotes cell migration and invasion of fibroblast-like synoviocytes by suppression of miR-27a in rheumatoid arthritis. Human Cell 2018; 31: 14-21.

50. Liao S, Zhou S, Wang C. GAPLINC is a predictor of poor prognosis and regulates cell migration and invasion in osteosarcoma. Biosci Rep 2018; 38: pii: BSR20181171.

51. Luo Y, Ouyang J, Zhou D, Zhong S, Wen M, Ou W, et al. Long Noncoding RNA GAPLINC Promotes Cells Migration and Invasion in Colorectal Cancer Cell by Regulating miR-34a/c-MET Signal Pathway. Dig Dis Sci 2018; 63: 890-9.

52. Mo BY, Guo XH, Yang MR, Liu F, Bi X, Liu Y, et al. Long Non-Coding RNA GAPLINC Promotes Tumor-Like Biologic Behaviors of FibroblastLike Synoviocytes as MicroRNA Sponging in Rheumatoid Arthritis Patients. Front Immunol 2018; 9: 702.
53. Liu H, Pope RM. The role of apoptosis in rheumatoid arthritis. Curr Opin Pharmacol, 2003; 3: 317-22.

54. Yan ZF, Zhao XY, Liu W, Liu XP. UCA1 impacts progress of rheumatoid arthritis by inducing the apoptosis of fibroblast-like synoviocyte. Eur Rev Med Pharmacol Sci 2018; 22: 914-20.

55. Yan ZF, Zhao XY, Liu W, Liu XP. UCA1 impacts progress of rheumatoid arthritis by inducing the apoptosis of fibroblast-like synoviocyte. Eur Rev Med Pharmacol Sci 2018; 22: 914-20.

56. Pan F, Zhu L, Lv H, Pei C. Quercetin promotes the apoptosis of fibroblast-like synoviocytes in rheumatoid arthritis by upregulating IncRNA MALAT1. Int J Mol Med 2016; 38: 1507-14.

57. Wang Y, Kong D. LncRNA GAS5 Represses Osteosarcoma Cells Growth and Metastasis via Sponging MiR-203a. Cell Physiol Biochem 2018; 45: 844-55.

58. Chen L, Yang W, Guo Y, Chen W, Zheng P, Zeng J, et al. Exosomal lncRNA GAS5 regulates the apoptosis of macrophages and vascular endothelial cells in atherosclerosis. PLoS One 2017; 12: e0185406.

59. Li G, Liu Y, Meng F, Xia Z, Wu X, Fang Y, et al. Tanshinone IIA promotes the apoptosis of fibroblast-like synoviocytes in rheumatoid arthritis by up-regulating lncRNA GAS5. Biosci Rep 2018; 38.

How to cite this article: Liang J, Chen W, Lin J. LncRNA: An allrounder in rheumatoid arthritis. J Transl Int Med 2019; 7: 3-9. 